\title{
GENFIRE: A Generalized Fourier Iterative Reconstruction Algorithm for High- Resolution 3D Electron and X-ray Imaging
}

\author{
Alan Pryor, Jr. ${ }^{1}$, Yongsoo Yang ${ }^{1}$, Arjun Rana ${ }^{1}$, Marcus Gallagher-Jones ${ }^{1}$, Jihan Zhou ${ }^{1}$, Yuan Hung Lo ${ }^{1}$, \\ Georgian Melinte ${ }^{2}$ Jose A. Rodriguez ${ }^{3}$, and Jianwei Miao ${ }^{1}$ \\ 1. Department of Physics and Astronomy and California Nanosystems Institute, University of California \\ Los Angeles, California, USA. \\ ${ }^{2}$ Institut de Physique et Chimie des Matériaux de Strasbourg, CNRS-Université de Strasbourg, \\ Strasbourg, France. \\ ${ }^{3 .}$ Department of Chemistry \& Biochemistry, UCLA-DOE Institute of Genomics and Proteomics, Los \\ Angeles, California, USA.
}

The 3D reconstruction of an object from a series of 2D projections is an important problem occurring in various fields across lengths ranging from millimeter-scale medical computed tomography (CT) to atomic electron tomography (AET) [1-4]. As the number of projection images that can be acquired is usually limited either by geometric constraints and/or tolerable radiation dose, the solution to the problem must provide the best-possible reconstruction from a limited number of projections. While most tomographic reconstruction methods are largely unchanged since the 1980s [4-6], the capabilities of imaging methods and technologies continue to advance [2,7-10]. To address the growth and comprehension of imaging modalities, we present a powerful tomographic reconstruction algorithm, termed GENFIRE (GENeralized Fourier Iterative REconstruction). In GENFIRE, a series of 2D projections are first converted to Fourier slices by the fast Fourier transform. Using a gridding method, the Fourier slices are assembled on to a Cartesian grid. The algorithm then iterates between real and reciprocal space, where physical constraints such as positivity and support are enforced in real space and the measured Fourier components are applied in reciprocal space. An error metric is monitored, and upon reaching suitable convergence the algorithm terminates and a high-quality reconstruction is obtained. GENFIRE produces reconstructions that are superior to several state-of-the-art tomographic reconstruction techniques [4-6,11]. To further improve the reconstruction, a refinement procedure can be used to correct the error in the angular measurement. GENFIRE will be made available freely and is equipped with a graphical user interface.

A number of numerical simulations, including biological samples as well as multislice simulation of nanocrystals, verify that GENFIRE produces reconstructions with higher resolution and less noise than other iterative tomographic methods [4-6,11]. Experimentally, ADF-STEM was combined with GENFIRE to reveal complex features of an FePt nanoparticle with unprecedented 3D atomic detail including chemical order/disorder, grain boundaries, anti-phase boundaries, anti-site point defects and swap defects [12].The full reconstruction quality reached in this experiment was not realized until GENFIRE was used both for reconstruction and identification of angular misalignments. GENFIRE has also been applied in correlative microscopy techniques to localize individual functionalized nanoparticles within mammalian cells combining ptychographic coherent diffractive imaging (CDI) with fluorescence and X-ray microscopy [13]. The availability of a generalized, multimodal 3D reconstruction algorithm in an open-source format with an easy-to-use graphical interface would be potentially transformative as interest in relevant fields of microscopy continues to grow. 


\section{References:}

[1] Kak, A. C. \& Slaney, M. Principles of Computerized Tomographic Imaging (SIAM, Philadelphia, 2001).

[2] J. Miao et al., Science 353 (2016), p. aaf2157.

[3] R. Fernandez-Leiro \& S. H. W. Scheres, Nature 537 (2016), p. 339.

[4] R. M. Mersereau and A. V. Oppenheim, Proc. IEEE, 62 (1974), p. 1319.

[5] R. Gordon, R. Bender, and G. T. Herman, J. Theor. Biol., 29 (1970), p. 471.

[6] A. Andersen, Ultrason. Imaging, 6 (1984), p. 81.

[7] J. Miao et al., Science 348 (2015), p. 530.

[8] M. C. Scott et al., Nature 483 (2012), p. 444.

[9] C. C. Chen et al., Nature 496 (2013), p. 74.

[10] R. Xu et al., Nature Mater. 14 (2015), p. 1099.

[11] J. Miao et al., Phys. Rev. B. 72 (2005), p.052103.

[12] Y. Yang et al., Nature 542 (2017), p. 75.

[13] M. Gallagher-Jones et al., submitted (2017).

Figure 1. Schematic layout of the GENFIRE algorithm.
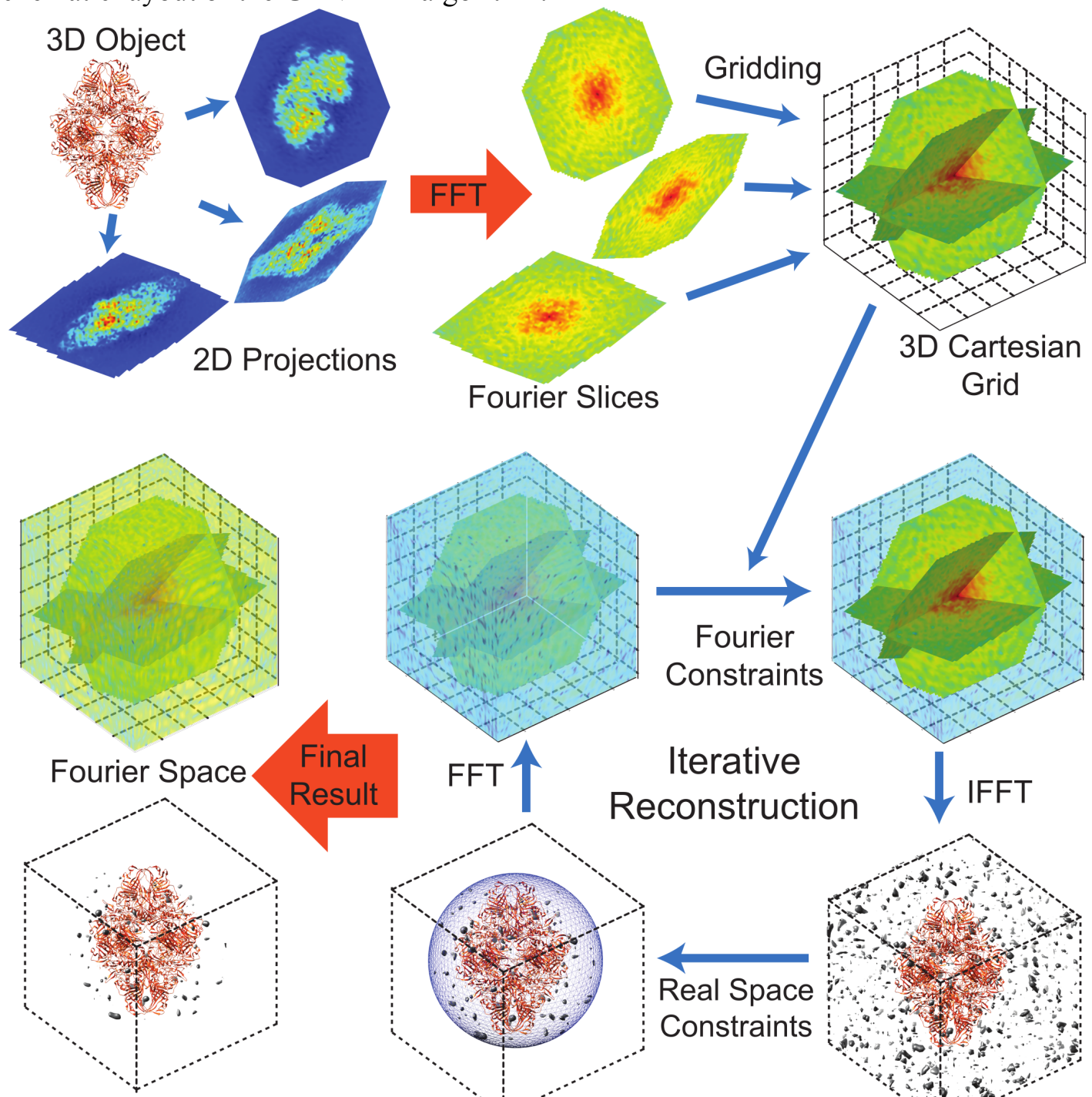

Real Space
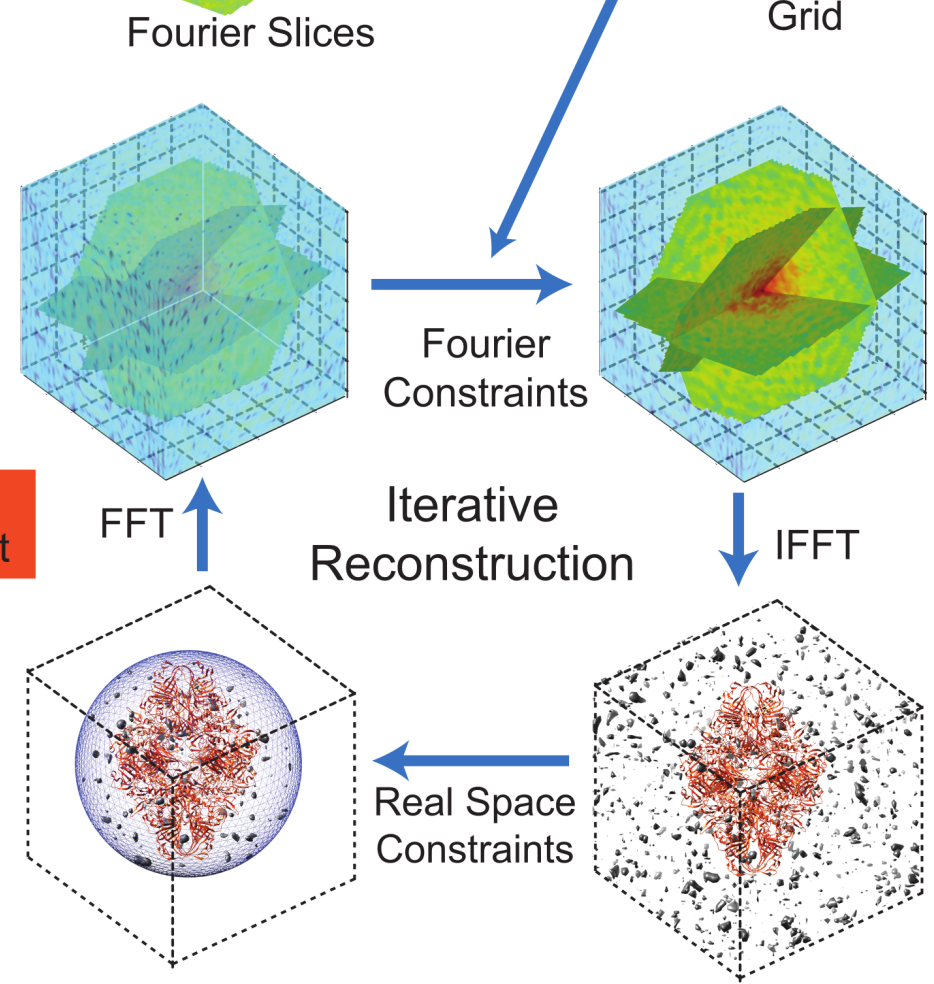

Real Space Constraints

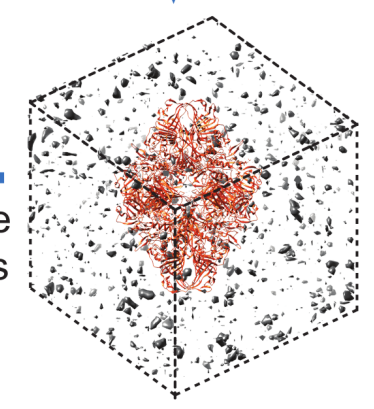

